\title{
Man with Malaria in Delaware
}

\author{
Benjamin Ngo, MD
}

\section{Case Presentation}

A 37-year-old African American male presented to the hospital complaining of high fevers over the past two weeks associated with severe posterior headache. He described having had a very poor appetite recently, but denied any cough, abdominal pain, diarrhea, or urinary symptoms. He did not recall sustaining any head trauma. He did relate, however, that he had recently returned from a three-month trip to Kenya $\sim 1$ month prior to this presentation. He had no past medical history and was not on any medications. He did not use IV drugs. Physical exam confirmed a fever of $102.1^{\circ} \mathrm{F}$ though normotensive $110 / 82 \mathrm{~mm}$ $\mathrm{Hg}$ and a resting heart rate $110 \mathrm{bpm}$. The complete physical exam including cardiopulmonary, abdominal, and neurological examination was normal. Laboratory analysis was unrevealing. A head CT performed prior to the lumbar puncture also failed to reveal any abnormalities. However, given the patient's recent travel history, the team empirically began him on antimalarial treatment against $P$. falciparum (quinine sulfate $10 \mathrm{mg} / \mathrm{kg}$ every eight hours and doxycycline $100 \mathrm{mg}$ po bid) while awaiting confirmatory histologic diagnosis. The next day malaria was confirmed when the results of the thick and thin smear were positive for plasmodium with a viral level greater than $1 \%$. During his hospitalization he was noted to have recurrent fevers occurring almost every other day. His clinical status remained stable without any alterations of his laboratory data. He was discharged to complete his course of oral medications.

\section{Discussion}

$\sim 300,000$ to 500,000 new cases of malaria occur worldwide with 700,000 to 2.7 million deaths estimated worldwide. $\sim 30,000$ travelers from industrialized nations countries reportedly contract malaria. Infection occurs through the transmission of an infected Anopheles mosquito. Human malaria is caused by four species of Plasmodia: P. falciparum, P. vivax, P. ovale, and $P$. malariae. Each of the four species causes clinical disease by resulting ultimately in hemolysis. $P$. falciparum has the ability to invade red blood cells of all ages and it is associated with the highest risk and rate of morbidity and mortality. Relapsing fevers remain the clinical hallmark of malaria. Anemia, thrombocytopenia, splenomegaly, hepatomegaly and jaundice can develop, and splenic rupture can occasionally occur as well. Altered consciousness, oliguira, hypoglycemia, and organ failure are characteristics of severe $P$. falciparum infection.

The diagnosis should be suspected in patients with recurrent fevers that travel to endemic areas. The gold standard of diagnosis is through examination of thick and thin smears with light microscopy. Parasite levels are measurable and levels can correlate with both type of species as well as portend overall morbidity. Microscopy is regularly employed to assess a patient's response to treatment. Other diagnostic tests available include antigen detection, fluorescent microscopy, PCR and serologic markers analysis. Thick and thin blood smears should be examined at least every 12 hours to monitor the efficacy of therapy until the parasitemia is below $1 \%$. Smears should also be obtained at 3,7 , and 28 days to rule out recurrence or incomplete clearance of parasitemia.

There are several medications available for the treatment of malaria including quinoline derivatives, antifolates, artemisinin derivatives, and antimicrobials. $P$. ovale and $P$. malariae are universally sensitive to chloroquine. Several strains of chloroquine-resistent $P$. vivax have been reported. The Centers for Disease Control (CDC) recommend treatment of chloroquine sensitive species of malaria with chloroquine followed by a course of primaquine. Resistant $P$. vivax can be treated with mefloquine or quinine sulfate plus doxycycline. Clinically, it is very important that one distinguish between $P$. falciparum and the other species of malaria, as $P$. falciparum is largely resistant to chloroquine and this discernment will ultimately affect the final drug cocktail prescribed. Failure to identify P. falciparum could otherwise result in inadequate and delayed treatment with the wrong medications. Fortunately, many treatment regimens exist for the treatment of $P$. falciparum. The most commonly recommend regimen is quinine sulfate with pyrimethaminesulfadoxine or doxycycline.

When advising patients on foreign travel, physicians should be aware of resistance patterns of malaria based upon geographic locale. This will tailor the choice of malarial prophylaxis unique to the area. Prevention is paramount for travelers to endemic areas and many resources through the CDC exist to provide education to both clinicians and patients on anti-malarial drugs.

\section{References}

1. Kain, KC. Malaria in travelers. Epidemiology, disease, and prevention. Infectious Disease Clinics of North America 1998; 12:267.

2. Filler, S, Causer, LM, Newman, RD, et al. Malaria surveillance--United States, 2001. MMWR Surveillance Summary 2003; 52:1

3. Greenwood, BM et al. Malaria. Lancet 2005; 365:1487.

4. White, NJ. The treatment of malaria. NEJM 1996; 335:800. 